\title{
Materiales y técnicas para el acoplamiento mecánico óptimo de piezocerámicas en aire
}

\author{
T. E. GÓMEZ ÁlVAREZ-ARENAS , F. MONTERO DE ESPINOSA \\ Instituto de Acústica CSIC, Serrano 144, 28006 Madrid
}

\begin{abstract}
En los últimos años se están realizando grandes esfuerzos para poder realizar inspecciones de Ensayos no Destructivos por ultrasonidos sin emplear líquidos como medio de acoplo entre los transductores piezoeléctricos y las estructuras a inspeccionar. En este campo compiten dos tecnologías de transducción, los transductores basados en piezocerámicas y los de tipo capacitivo. En el caso de los piezocerámicos, el punto crítico lo constituye la desadaptación de impedancias entre el resonador piezoeléctrico y el aire. Para soslayar este problema es posible utilizar capas de adaptación $(\lambda / 4)$. Para esto es necesario utilizar materiales con muy baja densidad y velocidad de propagación (impedancia acústica específica del orden de 0.1 MRayl), baja atenuación y buena sintonización a la frecuencia de trabajo. Con frecuencia, todas estas propiedades son extremadamente difíciles de conjugar. En el trabajo se presenta la caracterización de materiales con elevada porosidad y su utilización como líneas de transmisión $\lambda / 4$ para frecuencias en el entorno de $1 \mathrm{MHz}$. La caracterización - constantes elásticas y coeficiente de atenuación- se realiza con acoplamiento en aire. El uso de estos materiales como línea de adaptación logra mejoras de transmisión de hasta $35 \mathrm{~dB}$ y sensibilidad en modo emisión-recepción de hasta $-20 \mathrm{~dB}$.
\end{abstract}

Palabras clave: Transductores piezoeléctricos, Materiales porosos, ultrasonidos en aire, capas de adaptación de impedancias.

\section{Techniques and materials for optimum mechanical matching of piezoceramics vibrating in air}

Great efforts have been made over the last years to avoid the use of coupling agents between ultrasonic transducers and sample materials for NDE applications. Two different technologies compite for this particular application: piezoelectric and electrostatic transducers. In the first case, main problem is the huge impedance mismatch between the air and any piezoceramic. This can be partially solved using $\lambda / 4$ matching layers. Low density and slow velocities of sound propagation in the materials (acoustic impedance as low as 0.1 MRayl), low attenuation of ultrasonic waves and good tuning of the $\lambda / 4-$ resonance to the working frequency of the ceramic are requiered. Usually, all this requirements are extremely difficult to achive at once. It is presented, in this work, the mechanical characterisation of highly porous materials to be used as $\lambda / 4-$ matching layers at frequencies around $1 \mathrm{MHz}$. This characterisation (both elastic constants and attenuation) is performed using airborne ultrasonic waves. The use of these materials as matching layers for piezoelectric transducers in pitch-cath mode increases the transmision by $35 \mathrm{~dB}$ and provides sensitivities about $-20 \mathrm{~dB}$

Keywords: Piezoelectric transducers, porous materials, air-coupled ultrasound, impedance matching layers.

\section{INTRODUCCIÓN}

Durante los últimos años, las aplicaciones de los ultrasonidos que conllevan la propagación de estos a través del aire, están creciendo de forma considerable $(1,2)$. Estas son aplicaciones convencionales (médicas, ensayos no destructivos -END-, caracterización de materiales o microscopía acústica de barrido) en las que la etapa de acoplamiento mecánico entre el transductor piezoeléctrico y el material a inspeccionar (contacto directo mediante un gel, o bien acoplamiento por medio de un chorro de agua o por inmersión en agua) se suprime. Esto supone una notable simplificación de la tecnología, pero introduce un grave problema que es debido a la fuerte desadaptación entre las impedancias acústicas específicas del transductor piezoeléctrico y el aire.

La impedancia acústica específica de un medio se define como el producto de la densidad por la velocidad de propagación de las ondas longitudinales. Para el aire, la impedancia acústica específica toma un valor en torno a $410 \mathrm{Kg} / \mathrm{m}^{2} \mathrm{~s}$, mientras que para una cerámica piezoeléctrica tenemos valo- res en torno a $30 \times 10^{6} \mathrm{Kg} / \mathrm{m}^{2} \mathrm{~s}$. Esta fuerte desadaptación de impedancias entre el aire y cualquier cerámica piezoeléctrica tiene dos efectos negativos sobre el transductor cuando se intenta operar en aire (emitir o recibir señales ultrasónicas al aire o desde el aire respectivamente):

La transferencia de energía de la cerámica al aire o viceversa es extraordinariamente baja (pérdidas el orden de $-90 \mathrm{~dB}$ ).

La banda de respuesta en frecuencia del transductor es muy estrecha.

Esto produce que los sistemas de inspección por ultrasonidos en aire basados en transductores piezoeléctricos posean muy baja sensibilidad y respuesta en frecuencia altamente monocromática.

Para salvar este problema compiten dos tecnologías diferentes. La primera consiste en emplear transductores capacitivos [3], que poseen alta sensibilidad y respuesta muy ancha en frecuencia, aunque son inestables en el caso de los transductores capacitivos convencionales o de elevado coste como 
los transductores micromecanizados. La otra tecnología consiste emplear cerámicas piezoeléctricas y mejorar su adaptación al aire. Para esto se emplean varios procedimientos, que pueden ser complementarios:

Reducción de la impedancia acústica del transductor mediante la producción de composites piezoeléctricos (fundamentalmente composites de geometría 1-3: pilares cerámicos embebidos en una matriz plástica) $[4,5]$.

Interposición de una o varias capas cuarto de onda entre la cerámica y el aire [5].

Este trabajo se centra en el diseño de capas de adaptación para cerámicas piezoelectricas para aire y en los procedimientos de caracterización de materiales para este fin.

Una capa cuarto de onda es una capa de material que se coloca sobre la superficie radiante del transductor. Se diseña de tal forma que su primera (o incluso algún orden mayor) resonancia mecánica (modo propio asociado a su espesor) se encuentre dentro de la banda de frecuencia del transductor. La impedancia acústica específica se debe elegir de forma que la cantidad de energía radiada (para trabajar en modo emisor) o recibida (modo receptor) se maximize. Un criterio aproximado para cumplir esta condición es:

$$
Z_{c a}=\sqrt{Z_{1} Z}
$$

donde $Z$ es la impedancia acústica específica y los subíndices $c a, 1$ y 2 se refieren a: la capa de adaptación, y los dos medios a adaptar respectivamente (en este caso aire y una cerámica piezoeléctrica)

\section{CRITERIOS PARA EL DISEÑO DE CAPAS DE ADAPTACIÓN. RESULTADOS TEÓRICOS.}

Todos los resultados que se presentan en esta sección son cálculos teóricos. Estos se obtienen de considerar una cerámica piezoeléctrica (PZT-5A) vibrando en modo espesor y cuyas dimensiones laterales son mucho más grandes que el espesor. La misma hipótesis se imponen a las capas de adaptación que se añaden al transductor. El problema se resuelve imponiendo condiciones de contorno (continuidad de tensiones y desplazamientos) en todas las caras. Se calculan los campos de desplazamientos y tensiones y también los campos eléctricos (en el caso de la cerámica piezoeléctrica). En particular, los casos que se presentan son los correspondientes al modo "receptor", es decir, se considera una perturbación mecánica incidente en la cerámica y se calculan tensiones, desplazamientos y campo eléctrico en la cerámica. De ahí se obtiene la diferencia de potencial entre bornes de la cerámica.

\subsection{Impedancia acústica específica.}

Con frecuencia el único criterio empleado en el diseño de capas de adaptación es el indicado anteriormente en (1). Para el caso de acoplar una cerámica piezoeléctrica al aire, el resultado es:

$\mathrm{Kg} / \mathrm{m}^{2} \mathrm{~s}$. La figura 1 presenta los resultados obtenidos de calcular la respuesta eléctrica de un transductor piezoeléctrico monolítico (discos de PZT-5A), de frecuencia central $1 \mathrm{MHz}$, trabajando en modo recepción en aire y sin considerar atenuación en la capa de adaptación. Se supone que recibe una excitación mecánica de 1 micra de amplitud a cada frecuencia. La resonancia (cuarto de onda) de la capa de adaptación está per-

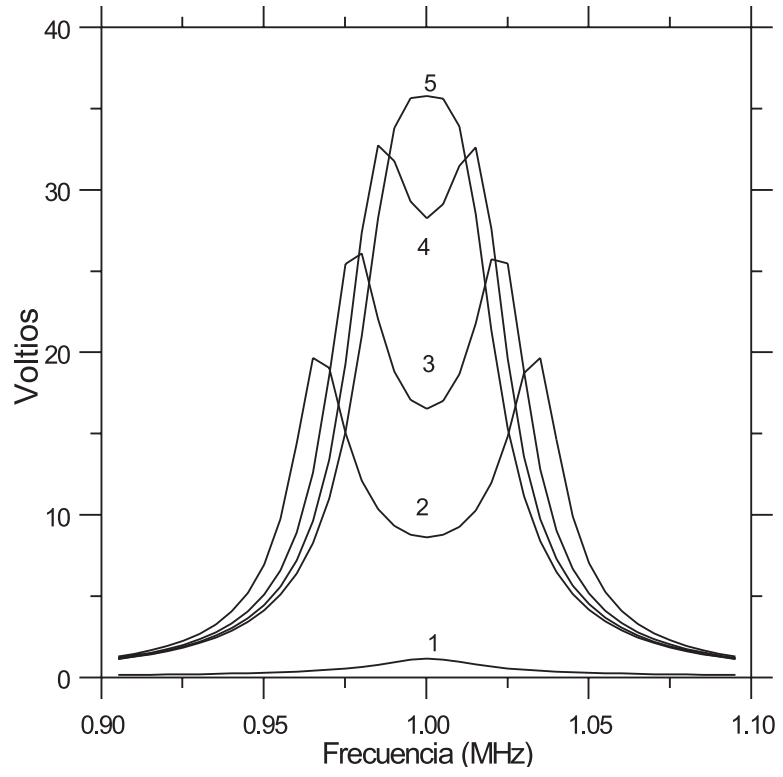

Figura 1. Amplitud de la respuesta eléctrica de una cerámica a una excitación mecánica de 1 micra de amplitud para diversos casos de capa de adaptación (impedancia de la capa): 1: sin capa de adaptación, 2: 0.2 MRayl, 3: 0.1 Mrayl, 4: 0.05 MRayl, 5: 0.025 MRayl.

fectamente sintonizada con la frecuencia del transductor. La mejor sensibilidad se obtiene para una impedancia de la capa de adaptación ente 0.025 y $0.1 \times 10^{6} \mathrm{Kg} / \mathrm{m}^{2} \mathrm{~s}$ sin presentar cambios muy acusados dentro de ese amplio margen de variación. No obstante, los resultados obtenidos para una capa de adaptación de impedancia $0.2 \times 10^{6} \mathrm{Kg} / \mathrm{m}^{2} \mathrm{~s}$ son también muy positivos. A medida que la impedancia acústica específica de la capa de adaptación se separa del rango ideal, la sensibilidad baja y el pico de resonancia se desdobla.

La primera dificultad en diseñar y fabricar capas de adaptación cuarto de onda para acoplar cerámicas piezoeléctricas para trabajar en aire reside en conseguir materiales que posean una impedancia acústica específica tan baja como la presentada anteriormente (entre 0.1 y $0.025 \times 10^{6} \mathrm{Kg} / \mathrm{m}^{2} \mathrm{~s}$ ). Para conseguir esos valores de la impedancia acústica específica no basta con emplear materiales muy ligeros (polímeros, plásticos, compuestos orgánicos, etc..) sino que además es necesario que presenten muy alta porosidad (porosidades típicas de entre 0.5 y 0.95). La tabla I muestra algunos de los materiales empleados para la fabricación de capas de adaptación.

TABLA I. PROPIEDADES básicAS DE ALGUNOS MATERIALES ÚtILES PARA LA FABRICACIÓN DE CAPAS DE ADAPTACIÓN PARA TRANSDUCTORES PIEZOELÉCTRICOS.

\begin{tabular}{|c|c|c|c|}
\hline Material & $\begin{array}{c}\text { Densidad } \\
\left(\mathrm{Kg} / \mathrm{m}^{3}\right)\end{array}$ & $\begin{array}{c}\text { Velocidad } \\
(\mathrm{m} / \mathrm{s})\end{array}$ & $\begin{array}{c}\text { Impedancia } \\
\left(10^{6} \mathrm{Kg} / \mathrm{m}^{2} \mathrm{~s}\right)\end{array}$ \\
\hline cona cargada con microesferas (1) & 500 & 600 & 0.3 \\
\hline Poliolefina microporosa (1) & 300 & 800 & 0.24 \\
\hline Nitrato de celulosa (2) & 360 & 343 & 0.123 \\
\hline Mezcla de nitrato y acetato de celulosa (2) & $450-580$ & $260-660$ & $0.117-0.383$ \\
\hline Papel (2) & $670-890$ & $300-700$ & $0.2-0.62$ \\
\hline Cartón (2) & 900 & 350 & 0.315 \\
\hline Aerogel (3) & $50-500$ & $50-500$ & $0.0025-0.25$ \\
\hline Metacrilato poroso (3) & $90-500$ & $290-600$ & $0.026-0.3$ \\
\hline
\end{tabular}

1: REFERENCIA 6 / 2: ReFERENCIA 7 / 3: ReFERENCIA 9 


\subsection{Sintonización de la capa de adaptación: Multicapas.}

Como es bien conocido y ya se ha mostrado (ver figura 1) los transductores piezoeléctricos operando en aire presentan una banda de respuesta en frecuencia muy estrecha. Por este motivo resulta imprescindible sintonizar muy bien la resonancia de la capa de adaptación a la resonancia de la cerámica. La figura 2 muestra la respuesta eléctrica calculada para un transductor piezoeléctrico operando en aire en modo receptor para distintas capas de adaptación, de impedancia $0.025 \mathrm{Kg} / \mathrm{m}^{2} \mathrm{~s}$, pero cuyo espesor no esta bien sintonizado a la frecuencia de trabajo de la cerámica. La resonancia de los transductores está situada a $1 \mathrm{MHz}$. La influencia de la sintonización de la capa cuarto de onda es fundamental. Una desviación del espesor del $1 \%$ puede producir caídas en la respuesta del transductor en un factor 3.5 además de introducir una notable distorsión de la banda de respuesta.

Este problema es más grave aún si se tiene en cuenta que, como ya se ha mencionado, los materiales que se necesitan son altamente porosos, por lo que es extremadamente difícil mecanizarlos para conseguir el espesor deseado. Esto significa que, en la gran mayoría de las veces, el material debe ser fabricado al espesor deseado, lo cual limita grandemente la disponibilidad del material. Para los datos mostrados en la tabla I, una capa de adaptación sintonizada a $1 \mathrm{MHz}$ debe tener un espesor que oscila entre 0.05 y $0.2 \mathrm{~mm}$. Esto supone, que la fabricación de capas de adaptación para transductores piezoeléctricos operando en aire para frecuencias en el rango de los megaherz implica la preparación de membranas delgadas y muy porosas.

El problema de la sintonización de la capa de adaptación se puede relajar si se emplea una capa de adaptación intermedia entre la cerámica y la membrana porosa. $\mathrm{Al}$ aumentar el número de capas cuarto de onda que se colocan sobre el transductor, la banda de respuesta en frecuencia se ensancha, aunque no se consigue mejora de la sensibilidad. El efecto de ensanchamiento de la banda rebaja considerablemente la restricción de la buena sintonización de la membrana porosa a la frecuencia del transductor.

Resultados similares a los mostrados en la figura 2 se muestran en la figura 3, pero en este caso, se inserta una capa de polímero entre la membrana porosa y la cerámica piezoeléctrica. Puede observarse como variaciones en la frecuencia de resonancia de la capa de adaptación de hasta un $20 \%$ no tienen efecto apreciable en la amplitud de respuesta del transductor, aunque sí modifican la frecuencia de resonancia.

\subsection{Pérdidas en la capa de adaptación.}

El uso de materiales porosos para confeccionar las capas de adaptación es obligado debido a los valores de densidad y velocidad de propagación (esto es, impedancia) que es necesario alcanzar. El principal problema surge del hecho de que este tipo de materiales presentan valores altos y muy altos de la atenuación. Scattering debido a los poros, relajaciones térmicas y viscoelásticas, inhomogeneidades en el material, son algunas de las fuentes de absorción. La situación es especialmente crítica para trabajar a alta frecuencia (por encima de $0.5 \mathrm{MHz}$ ) ya que la atenuación aumenta al menos linealmente con la frecuencia en este tipo de materiales. Por este motivo es fundamental medir siempre la atenuación en el material con el que se pretende realizar la capa de adaptación a la frecuencia de trabajo de la cerámica piezoeléctrica que se desea acoplar al aire. En ocasiones se da el caso de que aunque el material posee la

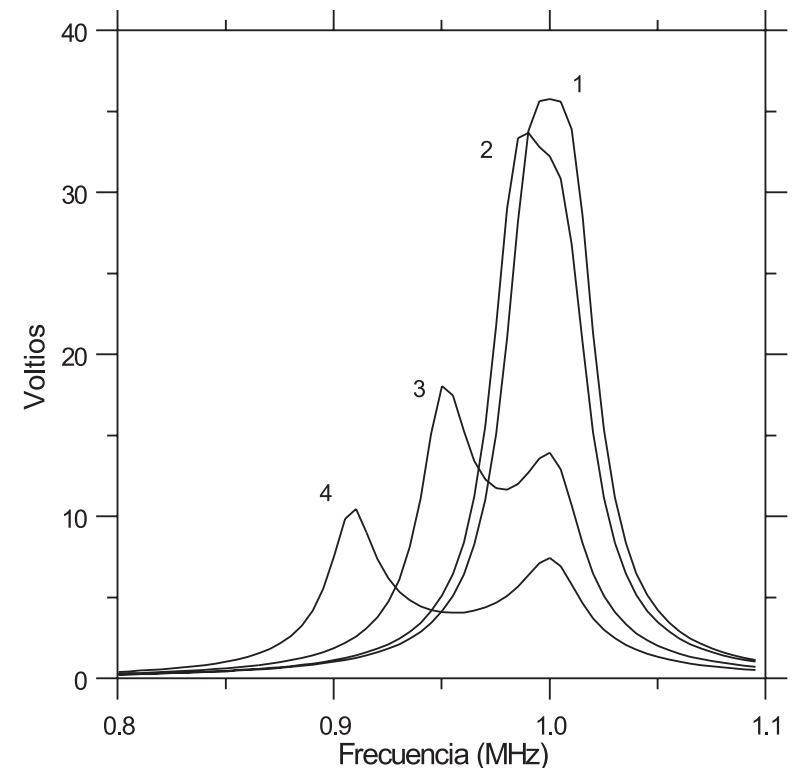

Figura 2.Amplitud de la respuesta eléctrica de un transductor piezoeléctrico $(1 \mathrm{MHz})$ con capa de adaptación cuarto de onda (tipo membrana porosa 0.025 MRayl) a una excitación mecánica de 1 micra de amplitud. La capa de adaptación se sintoniza a: 1: $1 \mathrm{MHz}$ (sintonización ideal); 2: $0.99 \mathrm{MHz}$ (1\%); 3: $0.95 \mathrm{MHz}(5 \%)$; 4: $0.9 \mathrm{MHz}(10 \%)$

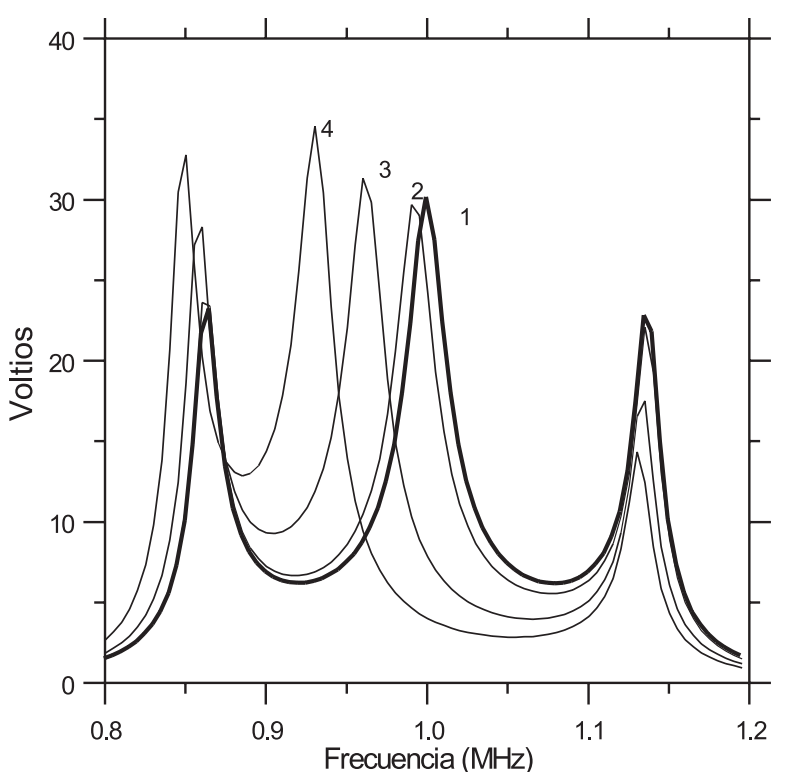

Figura 3. Amplitud de la respuesta eléctrica de un transductor piezoeléctrico $(1 \mathrm{MHz})$, a una excitación mecánica de 1 micra de amplitud, con una capa de adaptación polimérica (impedancia 2.75 Mrayl) otra tipo membrana porosa (0.025 MRayl) sintonizada a la frecuencia: 1: $1 \mathrm{MHz}$ (sintonización ideal); 2: $0.99 \mathrm{MHz}(1 \%)$; 3: $0.95 \mathrm{MHz}(5 \%)$; 4 : $0.9 \mathrm{MHz}(10 \%)$ 
TABla II. Propiedades bÁSICAS DE ALGUNOS MATERIALES PARA LA FABRICACIÓN DE CAPAS DE ADAPTACIÓN

\begin{tabular}{|c|c|c|c|}
\hline Material & $\begin{array}{c}\text { Impedancia } \\
\left(10^{6} \mathrm{Kg} / \mathrm{m}^{2} \mathrm{~s}\right)\end{array}$ & $\begin{array}{c}\text { Velocidad } \\
(\mathrm{m} / \mathrm{s})\end{array}$ & $\begin{array}{c}\text { Atenuación } \\
\text { frecuencia } \\
(\mathrm{Np} / \mathrm{m} / \mathrm{MHz})\end{array}$ \\
\hline $\begin{array}{c}\text { Silicona cargada con } \\
\text { microesferas }\end{array}$ & 0.3 & 600 & $1150 / 1$ \\
\hline Poliolefina microporosa & 0.24 & 800 & $345 / 1$ \\
\hline Nitrato de celulosa & 0.123 & 343 & $1055 / 1.42$ \\
\hline Aerogel & 0.0025 & 50 & 50 \\
& 0.25 & 500 & 100 \\
\hline
\end{tabular}
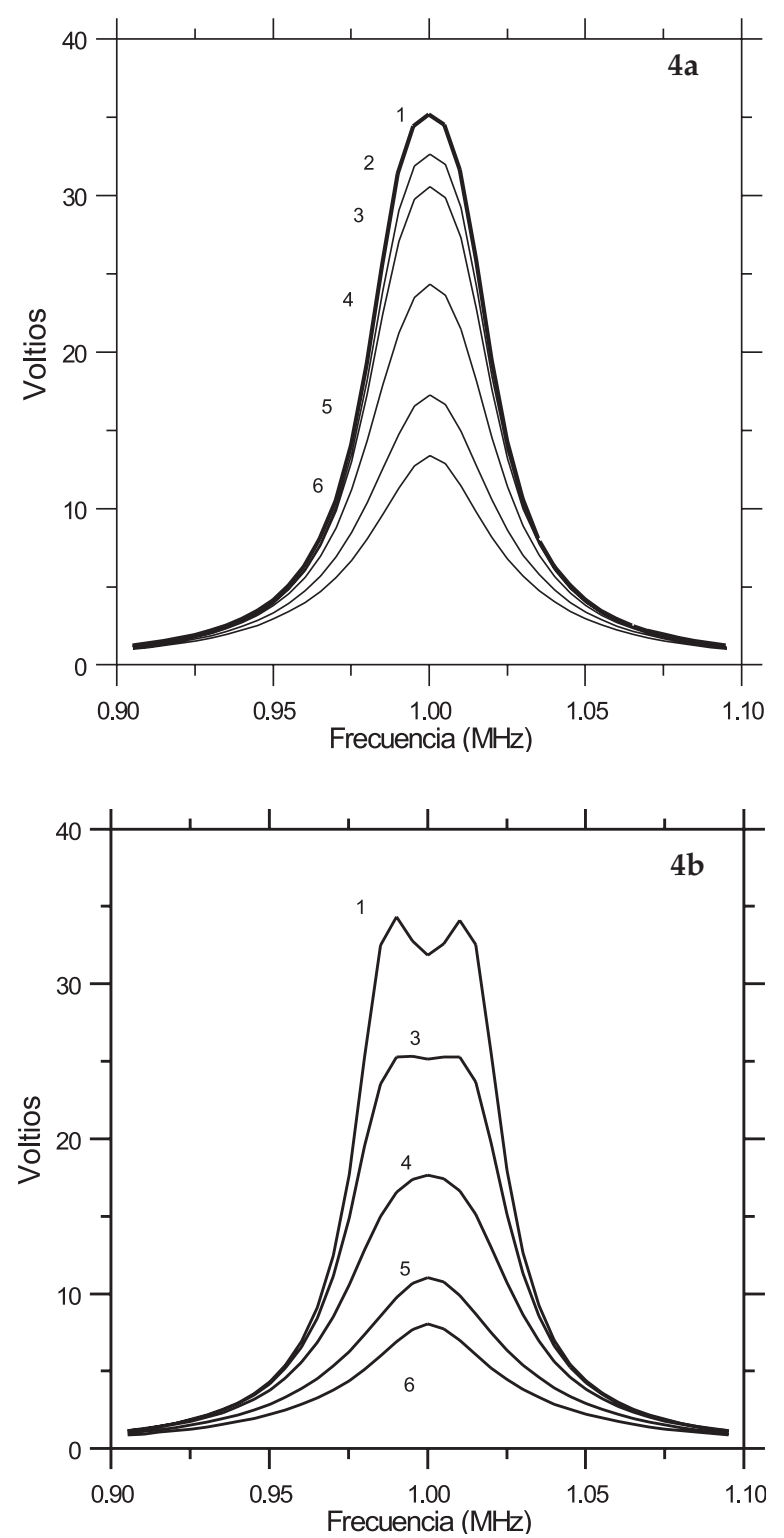

Figura 4. Amplitud de la respuesta eléctrica de un transductor piezoeléctrico $(1 \mathrm{MHz})$, a una excitación mecánica de 1 micra de amplitud, con una capa de adaptación tipo membrana porosa para los siguientes casos de atenuación en la membrana: 1: sin atenuación; $2: 50 \mathrm{~Np} / \mathrm{m}$; 3: $100 \mathrm{~Np} / \mathrm{m}$; 4: $300 \mathrm{~Np} / \mathrm{m} ; 5: 700 \mathrm{~Np} / \mathrm{m}$; 6: $1100 \mathrm{~Np} / \mathrm{m}$. Capa de adaptación: 4.a: v=200 m/s, 0.1 MRayl, espesor $50 \mu \mathrm{m}$. / 4.b:v=400 m/s, 0.4 MRayl, espesor $100 \mu \mathrm{m}$. impedancia acústica específica requerida, la atenuación es tan alta que el material resulta inútil como capa de adaptación. La tabla II muestra la atenuación de algunos de estos materiales.

La figura 4 muestra la amplitud de respuesta eléctrica de una cerámica piezoeléctrica (PZT-5A) con una capa de adaptación a una excitación mecánica monocromática de una micra de amplitud. Se emplean dos tipos distintos de membrana según se indica en la figura.

\section{CARACTERIZACIÓN DE MATERIALES.}

En esta sección se presenta una técnica de caracterización de materiales que permite obtener con precisión todos aquellos parámetros que influyen en su comportamiento como capa de adaptación para cerámicas piezoeléctricas actuando en aire. Estos son: Densidad, velocidad de propagación y atenuación de los ultrasonidos y calidad de la resonancia en modo espesor.

La técnica que se emplea es la de medir, a incidencia normal, el coeficiente de transmisión de una lámina del material a estudiar cuando incide sobre el una señal ultrasónica de banda ancha (propagación en aire) [7]. Para esto se emplean varias parejas de transductores piezoeléctricos de banda ancha que cubren el rango de medida: 0.3-2 MHz). El transductor emisor se excita con una señal eléctrica de alto voltaje y banda ancha. El transductor receptor recoge la señal, que luego es amplificada en $40 \mathrm{~dB}$, digitalizada y almacenada en un ordenador para su posterior tratamiento. La señal transmitida directamente del emisor al receptor se emplea para calibrar el sistema. Posteriormente, se coloca entre emisor y receptor la lámina de material a caracterizar, es necesario que dentro de la banda de frecuencia de los transductores se encuentre alguna resonancia asociada al espesor de la muestra (bien el primer orden o bien algún orden mayor). Las medidas se comparan con los cálculos teóricos para el coeficiente de transmisión a través de una lámina. La figura 5 muestra algunos de los resultados obtenidos, en particular TR (raíz cuadrada del coeficiente de transmisión) en función de la frecuencia). En todos los casos se observa la resonancia de primer orden de la lámina de material. De esta comparación teorico-experimental se obtienen: Velocidad de propagación y atenuación de los ultrasonidos y densidad del material.

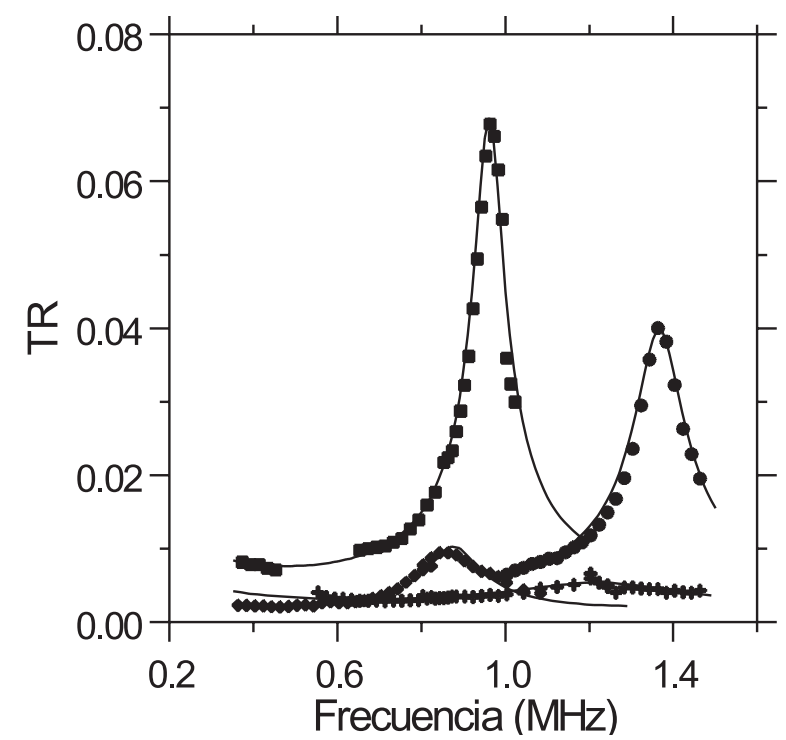

Figura 5.Raíz cuadrada del coeficiente de transmisión (TR) en función de la frecuencia para diferentes materiales porosos (ver tabla

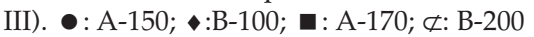


El método se empleó para caracterizar un buen número de materiales. La tabla III muestra los resultados de los que mejor se adecuaban a las restricciones que se imponen a una capa de adaptación para un transductor piezoeléctrico para aire. Los materiales tipo A (tabla III) son compuestos de celulosa muy porosos y de alta calidad (homogéneos y planoparalelos). Los materiales tipo B son distintos tipos de papel. En este caso, la homogeneidad y el planoparalelismo de las membranas no es tan alto como en el caso anterior. Claramente se puede ver como la atenuación del primer modo espesor es mucho más alta. Por lo que se refiere a la impedancia, los que más se aproximan al valor teórico son los materiales A-150 y A-170. Finalmente hay que indicar, que la primera resonancia para el coeficiente de transmisión de una lámina de material en aire está localizada en el valor que verifica la igualdad $t=\lambda / 2$ (siendo $\lambda$ la longitud de onda y $t$ el espesor de la lámina), mientras que la primera resonancia de esa misma lámina cuando se adhiere a la superficie de una cerámica, quedando la otra cara en contacto con el aire, tiene lugar cuando se verifica $t=\lambda / 4$. Es decir, que los materiales mostrados en la tabla III, funcionan como capas cuarto de onda para la frecuencia mitad a la indicada en la tabla.

\section{DISEÑO DE TRANSDUCTORES PARA OPERAR EN AIRE.}

Se tomaron capas de adaptación A-140 y A-170 para diseñar dos parejas de transductores. Una con frecuencia de trabajo en torno a $1.1 \mathrm{MHz}$ (A-140) y la otra en torno a $0.5 \mathrm{MHz}$ (A-170). En ambos casos se construyó un emisor y un receptor, ajustando las bandas de emisión y recepción (respectivamente) a la misma frecuencia, para evitar problemas de pérdidas por desajuste de las bandas. Los transductores se colocaban enfrentados a una distancia de $2 \mathrm{~cm}$ y se medía la sensibilidad en función de la frecuencia. La sensibilidad se define como:

$$
S=20 \log \left(\frac{V r}{V o}\right)(d B)
$$

donde $V r$ es la diferencia de potencial eléctrico (valor de pico) entre los electrodos de la cerámica del transductor receptor y

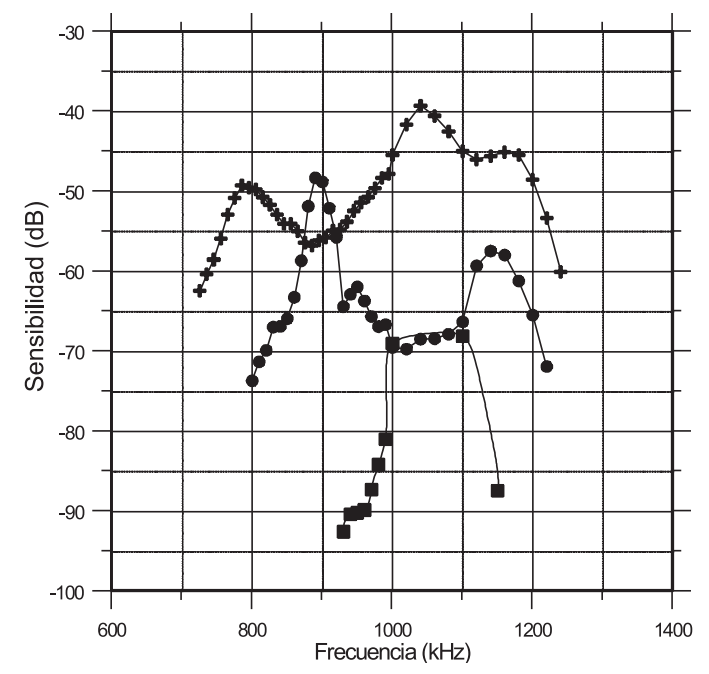

TABla III. Resultados DE LA CARACTERIZACIÓN DE MATERIALES PARA CAPAS DE ADAPTACIÓN.

\begin{tabular}{|l|l|l|l|l|l|}
\hline Material & $\begin{array}{l}\text { Velocidad } \\
(\mathrm{m} / \mathrm{s})\end{array}$ & $\begin{array}{l}\text { Densidad } \\
\left(\mathrm{Kg} / \mathrm{m}^{3}\right)\end{array}$ & $\begin{array}{l}\text { Impedancia } \\
\mathrm{Kg} /\left(\mathrm{s} \mathrm{m}^{2}\right)\end{array}$ & $\begin{array}{l}\text { Atenuación } \\
(\mathrm{Np} / \mathrm{m})\end{array}$ & $\begin{array}{l}\text { Frecuencia } \\
\lambda / 2(\mathrm{MHz})\end{array}$ \\
\hline \hline A-110 & 662 & 580 & 0.38 & $>3000$ & $\sim 3.6$ \\
\hline A-140 & 538 & 510 & 0.27 & $\sim 1500$ & -2.3 \\
\hline A-150 & 382 & 350 & 0.13 & 850 & 1.35 \\
\hline A-170 & 288 & 365 & 0.11 & 700 & 0.96 \\
\hline B-100 & 350 & 900 & 0.32 & 1100 & 0.9 \\
\hline B-200 & 300 & 890 & 0.27 & $>1500$ & 1.2 \\
\hline B-300 & 700 & 670 & 0.47 & $\sim 1500$ & $\sim 1.82$ \\
\hline
\end{tabular}

Vo es la diferencia de potencial eléctrico (valor de pico) aplicado a la cerámica del transductor emisor.

La sensibilidad se midió para los siguientes casos:

- Transductores sin capa de adaptación.

- Transductores con una capa de adaptación (A-140 o A170 según frecuencia)

- Transductores con una capa de adaptación de resina epoxy $\left(\mathrm{v}=2700 \mathrm{~m} / \mathrm{s}\right.$ densidad $\left.=1300 \mathrm{Kg} / \mathrm{m}^{3}\right)$

- Transductores con dos capas de adaptación:

Una primera de resina epoxy

Una segunda de membrana porosa A-140 o A-170 según frecuencia de trabajo.

Los resultados se muestran en la figura 6.a (1 MHz). y 6.b. (0.5 MHz) Puede observarse con claridad cómo aumenta la sensibilidad cuando se coloca una capa de adaptación (25-40 dB comparado con la situación en la que no se coloca capa de adaptación). Que la sensibilidad sea ligeramente mayor a la frecuencia de $0.5 \mathrm{MHz}$ puede relacionarse con una menor atenuación del aire a esa frecuencia. También se observa que las cerámicas, sin capa de adaptación, proporcionan una respuesta en frecuencia de banda muy estrecha, y que esta situación es claramente mejorada al colocar un apilamiento de, al menos, dos capas de adaptación. Estos transductores pueden usarse para análisis de superficies de sólidos o para inspecciones del interior de los materiales [8].

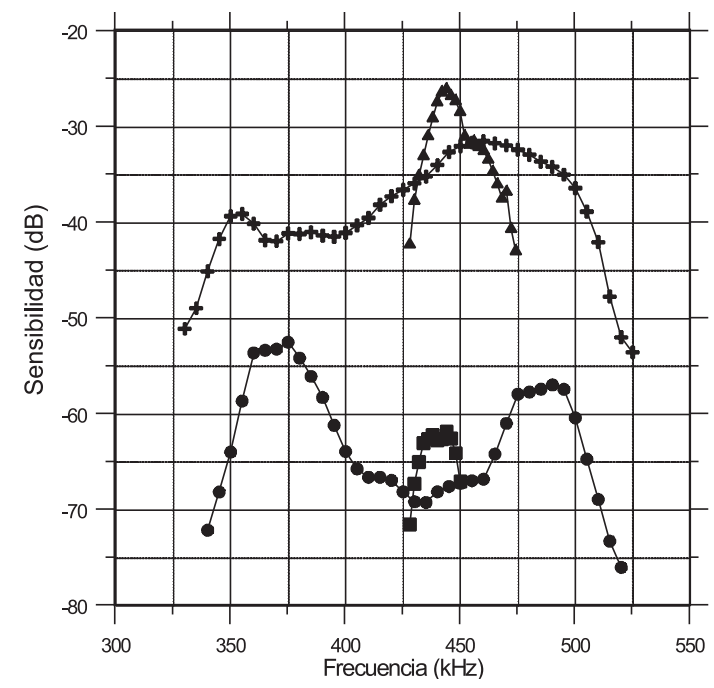

Figura 6. Sensibilidad de una pareja de transductores operando en modo emisor-receptor separados $2 \mathrm{~cm}$ en aire. Figura 6.a: frecuencia central $1 \mathrm{MHz}$, figura 6.b. Frecuencia central 0.5 MHz. $\mathbf{a}$ : Sin capa de adaptación; $\bullet$ : Con una capa de adaptación (resina epoxy); $\square$ : Con una capa de adaptación (membrana microporosa); $\mathbf{\Lambda}$ : Con dos capas de adaptación: resina epoxy y membrana microporosa. 


\section{CONCLUSIONES.}

Se ha desarrollado un estudio teórico para mostrar cuáles son los criterios para diseñar de forma óptima capas de adaptación cuarto de onda para acoplar mecánicamente cerámicas piezoeléctricas al aire. En segundo lugar se presenta un método de caracterización de materiales que permite determinar con precisión las magnitudes requeridas para el diseño de capas de adaptación. Se presentan los resultados obtenidos para algunos de los materiales que mejor cumplen con lo requisitos de diseño establecidos en la sección anterior. Finalmente se diseñan y construyen dos parejas de transductores piezoeléctricos para operar en aire: una pareja a $0.5 \mathrm{MHz}$ y la otra a $1 \mathrm{MHz}$. Se aplican, como capas de adaptación, algunos de los materiales encontrados y caracterizados previamente. Se consiguen sensibilidades (en modo emisión recepción) de hasta $-25 \mathrm{~dB}$. Con estas capas de adaptación, es posible emplear cerámicas piezoeléctricas para construir transductores para generación y recepción de señales ultrasónicas en aire.

\section{BIBLIOGRAFÍA}

1. V. Mágori. "Ultrasonic sensors in air" 1994 IEEE Ultrasonic Symposium. Proceedings 471-481, (1994).

2. W. A. Grandia, C. M. Fortunko “NDE applications of air-coupled ultrasonic transducers" 1995 IEEE Ultrasonic Symposium Proceedings 697-709, (1995)

3. H. Carr, C. Wykes "Diagnosis measurement in capacitive transducers" Ultrasonics 1[31], 13-20, (1993).

4. D. Reilly, G. Hayward "Through air transmission for ultrasonic non-destructive testing" 1991 IEEE Ultrasonic Symposium Proceedings 937-939, (1991)

5. M. I. Haller, B. T. Khuri-Yakub "1-3 composites for ultrasonic air transducers" 1992 IEEE Ultrasonic Symposium Proceedings 937-939, (1992)

6. T. Yano, M. Tone, A. Fukumoto. "Range finding and surface characterization using high-frequency air transducers". IEEE Trans. Ult. Ferroelec. and Freq. Ctrl, 34 [2], 232-236, (1987)

7. T. E. Gómez, F. Montero "Bridging the gap of acoustic impedance mismatch between air and solid materials" 2000 IEEE Ultrasonic Symposium. Proceedings 1069-1072, (2000)

8. F. Montero, T.E. Gómez, A. Albareda, R. Pérez. “High-sensitivity piezoelectric transducers for NDE air-borne applications" 2000 IEEE Ultrasonic Symposium. Proceedings, 1073-1076 (2000)

9. T.E. Gómez, F. Montero. E. Molins, R. Rodriguez “Low-impedance and Lowloss Customized Materials for Air-coupled Piezoelectric Transducers" 2001 IEEE Ultrasonic Symposium. Proceedings, (2001)

Recibido: 31.05.01

Aceptado: 10.12.01 